\title{
Towards Natural Non-Invasive Hand Neuroprostheses for Daily Living
}

\author{
Michele Tavella, Robert Leeb, Rüdiger Rupp, and José del R. Millán
}

\begin{abstract}
In this paper we show how healthy subjects can operate a non-invasive asynchronous BCI for controlling a FES neuroprosthesis and manipulate objects to carry out daily tasks in ecological conditions. Both, experienced and novel subjects proved to be able to deliver mental commands with high accuracy and speed. Our neuroprosthetic approach relies on a natural interaction paradigm, where subjects delivers congruent MI commands (i.e., they imagining a movement of the same hand they control through FES). Furthermore, we have tested our approach in a common daily task such as handwriting, which requires the user to split his/her attention to multitask between BCI control, reaching, and the primary handwriting task itself. Interestingly, the very low number of erroneous trials illustrates how during the experiments subjects were able to deliver commands just when they intended to do so. Similarly, the subjects could perform actions while delivering, or preparing to deliver, mental commands.
\end{abstract}

Index Terms-BCI, EEG, FES, neuroprosthetics, grasping, natural interaction, multitasking, manipulation.

\section{INTRODUCTION}

Handicapped persons suffering from the loss of hand grasping functionality need constant assistance for carrying on simple tasks of their daily living [1]. Nowadays, the most effective technique to elicit the contraction of paralyzed upper limb muscles is functional electrical stimulation (FES) by means of surface electrodes [2]. Commonly, FES systems for grasp restoration are controlled by residual muscular activity (e.g., movements of the contralateral shoulder). Thus normals users have spinal cord lesions below C5. For lesions of higher spinal cord segments, which leads to an extended loss of upper extremity function, other means for controlling the neuroprosthesis have to be considered.

The novel communication channel provided by a braincomputer interface $(\mathrm{BCI})$ has proven to be an effective way for controlling assistive technology (AT) devices, such as virtual keyboards [3] and even intelligent wheelchairs [4]. For this reason, non-invasive BCIs, based on electroencephalogram (EEG) signals, have been successfully coupled with FES neuroprostheses to restore whole hand prehension in a tetraplegic patient who control the neuroprosthesis by means of feet motor imagery (MI) to trigger sequential commands to open and close his paralyzed hand [5], [6].

This research is supported by the European ICT Programme Project FP7224631 (TOBI). This paper only reflects the author's views and funding agencies are not liable for any use that may be made of the information contained herein.

M. Tavella, R. Leeb and J.d.R. Millán are with the Chair on Non-Invasive Brain-Machine Interface, Center for Neuroprosthetics, School of Engineering, Ecole Polytechnique Fédérale de Lausanne, Lausanne, Switzerland, michele.tavelladepfl.ch jose.millandepfl.ch

R. Rupp is with the University Hospital, Spinal Cord Injury Center, Heidelberg, Germany.
In this paper we show how a non-invasive neuroprosthesis can be used in combination with natural interaction paradigms to regain the functionality of grasping and manipulation of objects. In contrast with the current state of the art, we propose an approach in which the subject is imagining a movement of the same hand he controls through FES. Furthermore, the performance of the user is evaluated in a handwriting task, which resembles a typical daily activity requiring the user to split his attention between BCI control and the task itself. In other words, to achieve the goal the subject must do multitasking, simultaneously controlling the $\mathrm{BCI}$ and executing another primary task.

\section{METHODS}

\section{A. Brain-Computer Interface}

Subjects delivered mental commands through an asynchronous spontaneous EEG-based BCI [7], [8]. To control the $\mathrm{BCI}$, the user learned to voluntary modulate EEG oscillatory rhythms by performing motor imagery (MI) tasks (i.e., right/left hand or feet imagined movements) without the need for any external stimulus and/or cue.

EEG was recorded with a portable 16-channel g.tec system at $512 \mathrm{~Hz}$ and band-pass filtered between $0.1 \mathrm{~Hz}$ and $100 \mathrm{~Hz}$. Each channel was then spatially filtered with a Laplacian derivation before estimating its power spectral density (PSD) in the band $4-48 \mathrm{~Hz}$ with $2 \mathrm{~Hz}$ resolution over the last second. The PSD was computed every $62.5 \mathrm{~ms}$ (i.e., 16 times per second) using the Welch method with 5 overlapped $(25 \%)$ Hanning windows of $500 \mathrm{~ms}$.

To facilitate and speed up BCI control, we use machine learning techniques to select subject-specific spatiofrequency features that maximize the separability between the different mental tasks [8]. We select the features with high discriminant values consistently across the whole training set to train a statistical Gaussian classifier [7]. These are the features the user can naturally modulate. The classifier computes the probability distribution over the mental tasks of an EEG sample. The BCI integrates over time the outputs of the classifier until it accumulates enough evidence about the user's mental intent. To do so, the BCI first rejects classifier decisions that are below a confidence probability threshold. Then, the BCI accumulates the surviving decisions using an exponential smoothing probability integration framework until the probability of a class reaches a given threshold. At this moment the corresponding mental command is delivered and the probability distribution is reset to uniform distribution. 


\section{B. Functional Electrical Stimulation}

FES delivers short current impulses that elicit action potentials on the efferent nerves, thus causing muscle contractions. Our system is a Microstim8 (Krauth \& Timmermann, Germany) stimulator with biphasic, rectangular constant current pulses. The frequency of the stimulation was set to $20 \mathrm{~Hz}$ to ensure tetanic contraction, while the pulse widths of the different stimulation channels were controlled by the BCI to trigger the opening and the closing of the hand of the subject. Current amplitude was set on an individual basis.

To achieve an effective opening of the hand, we stimulated the fingers extensor muscle (Musculus extensor digitorum communis) to extend the medial four digits of the hand. Hand closing was achieved by stimulating the fingers flexor muscle (Musculus flexor digitorum superficialis). In the experiments reported in this paper subjects performed a power grasp, which was executed in two phases: hand (and thumb) opening and closing.

\section{Subjects and Experimental Paradigm}

A total of four right-handed healthy subjects (A6, B2, $\mathrm{B} 3$ and B4; 23-27 year-old) were trained to control our 2class BCI, using MI of the dominant hand vs. the other hand or feet. All subjects were able to deliver mental commands performing dominant hand MI, which was mapped to actions of the same hand elicited by FES. Such a mapping provides a more natural and direct interaction paradigm that helps the user in manipulating objects by mere thinking.

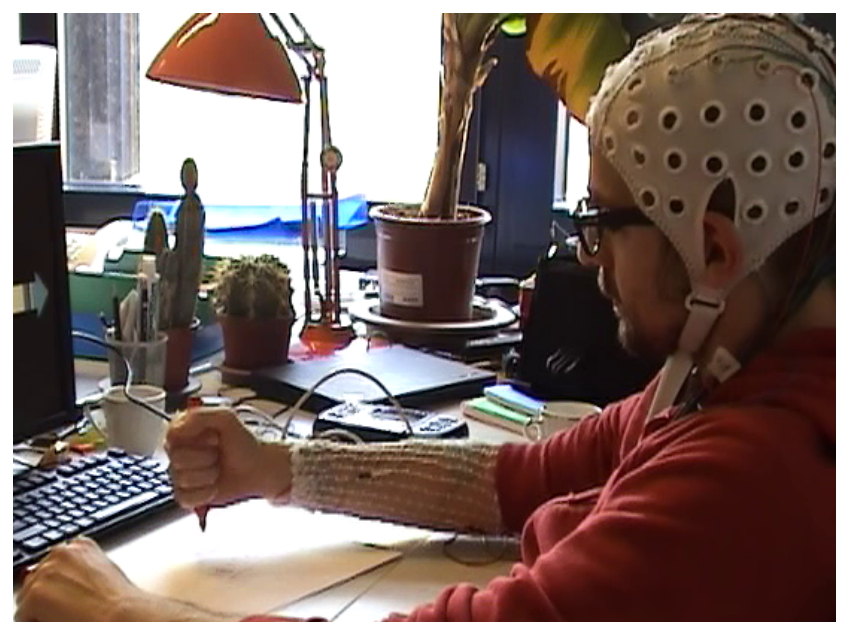

Fig. 1. Experimental setup: the subject holds the pen with a power grasp for writing a word. The FES electrodes are visible on his right forearm.

The task the subjects needed to accomplish was to mentally operate the FES to hold a pen with a power grasp for writing a word and releasing the pen once they finished (see Figure 1). A trial is defined as the sequence of grasping, writing and releasing (Figure 2). As mentioned above, the $\mathrm{BCI}$ delivers congruent MI commands to carry out grasping and releasing (i.e., imagined movements of the dominant hand where the FES are placed). Thus the MI command triggered FES patterns that depends on the status of the hand, either resting or active. If resting FES led to a power grasp (which is executed in two phases, hand opening and closing); if active because holding the pen, FES opened the hand.

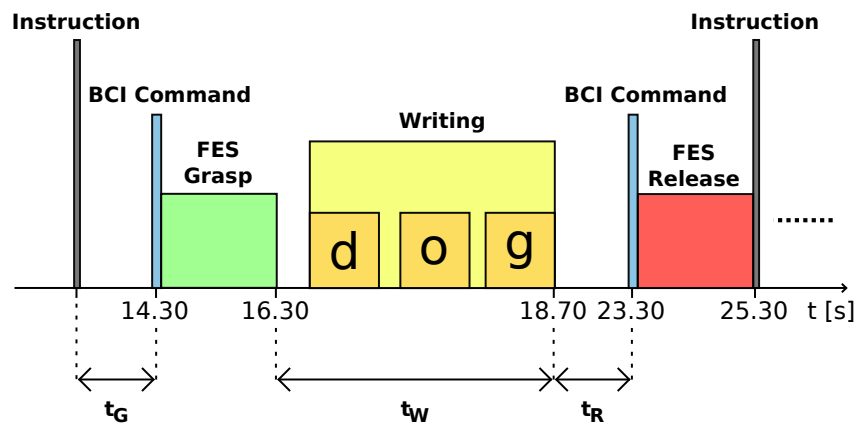

Fig. 2. The figure depicts an on-line trial in which the subject B3 had to write the word "dog" (time scale is not linear). He delivers congruent MI commands to the FES so as to grasp and release the pen ( $t_{g}$ and $t_{r}$, respectively). As soon as the subject released the pen, he is informed about the next task, which he must start as quickly as possible.

During the experiment the subject had to write 35 words or short sentences: 10 short words of 3-4 letters (e.g., "dog", "Oslo", "Rome"), 15 medium length words of 5-6 letters (e.g., "hello", "London", "Lisbon") and 10 short sentences of 13-16 letters (e.g., "The dog is kind", "My sister is tall", "The room is small"). These pieces of text were randomly split in 7 groups of 5 . Writing a group of 5 texts is a run and the 7 runs were performed sequentially. A failure in grasping, the premature release of the pen or the incorrect release of the pen in its holder are considered as errors. If an error happened during a trial, the trial stopped and was repeated from the beginning.

For each successful trial we measured the time needed to deliver the mental command for FES grasp, the time required to accomplish the writing task, and the time to send mental command for FES release. These three times are indicated as $t_{g}, t_{w}$ and $t_{r}$ in Figure 2, respectively.

Operating the neuroprosthesis for a handwriting task is an ecological activity that requires the user to split his/her attention between BCI control and the task itself. Actually, real handwriting requires the subject to $a$ ) reach for the pen (which is in a holder), $b$ ) grasp it, $c$ ) place the hand over the paper, $d$ ) write a word or short sentence in a sheet of paper in front of him/her, $e$ ) reach back the pen holder, $f$ ) release the pen in the holder, and $g$ ) bringing back the hand over the paper. Only at steps $b$ ) and $f$ ) the subject must deliver the mental command. Once the pen was released back in its holder, the operator instructed the subject about the next word or sentence to write, and another trial began. Note that the subject received the instruction of the next text while he was moving back the hand to the "home" position over the paper. By deploying an asynchronous paradigm, the subjects performed the task at their own pace, although they were asked to initiate it and to perform it as quickly as possible.

Even though preliminary tests with some of our subjects showed that they were able to write while the FES was active to sustain the power grasp, continuous stimulation may lead to rapid muscle fatigue preventing users to carry 
out manipulation tasks for long times. To cope with this limitation we have developed a passive hand orthosis that supports and synchronizes the movement of the fingers driven by FES [9]. More importantly, the orthosis is also equipped with a mechanical lock that holds fingers in the desired position (e.g., flexed in a grasp posture) without the need for continuous stimulation. Although the subjects were not wearing the hand orthosis during the experiments reported here, we switched off FES during writing (i.e., in the period $t_{w}+t_{r}$ in Figure 2).

As discussed, handwriting requires the user to multitask between BCI control, reaching, and the primary handwriting task itself. To further promote daily life conditions, the subjects were not asked to maintain a specific posture during the experiment. In fact, they were invited to behave naturally during the experiment and even to engage in verbal interaction with people around if they wished so. Moreover, while writing, the subjects were instructed not to perform the MI tasks (i.e., they could not engage in MI of the opposite $\mathrm{BCI}$ task to avoid triggering the FES).

\section{RESULTS}

Figure 3 shows the average and the standard deviation of the time required to the subjects to perform the grasp and release tasks by delivering MI commands to the asynchronous BCI. Referring to Figure 2, "grasp" time $t_{g}$ is the difference between the end of the previous trial (FES release) and the delivery of the BCI command for grasping, whereas "release" time $t_{r}$ is the difference between the end of writing the instructed text and the delivery of the BCI command for releasing. Thus, grasp time takes into account listening to the next text to write (and, simultaneously, moving the hand to the home position over the paper) and reaching for the pen holder, while release time also includes reaching for the pen holder and placing the pen in. The values are computed across the sets of short, medium and long texts. Although the time required to deliver the mental command depends on the ability of each subject in controlling the BCI, no subject exhibits a statistically significant difference in his/her grasp and release times across the three categories of text. Furthermore, it has to be mentioned that subject A6 and B4 were novel in controlling the $\mathrm{BCI}$, while $\mathrm{B} 2$ and $\mathrm{B} 3$ were experienced BCI users.

Figure 4 depicts the average and the standard deviation of the duration of the writing task $t_{w}$ (defined as the difference between the end of the FES grasp and the end of writing, Figure 2), for all the subjects and the three categories of text. As already mentioned, the subjects were asked to complete the writing task as quickly as possible, although no other suggestion was made. Thus, each subject spontaneously adopted his own strategy and handwriting style in accomplishing the task. For example, subject A6 did not consider the task completed until all the letters where perfectly readable, while subject B3 preferred to write in cursive, in contrast to all the other subjects who preferred block letters. Moreover, it is important to consider that the subjects were using power grasp to hold the pen and write. Although they were
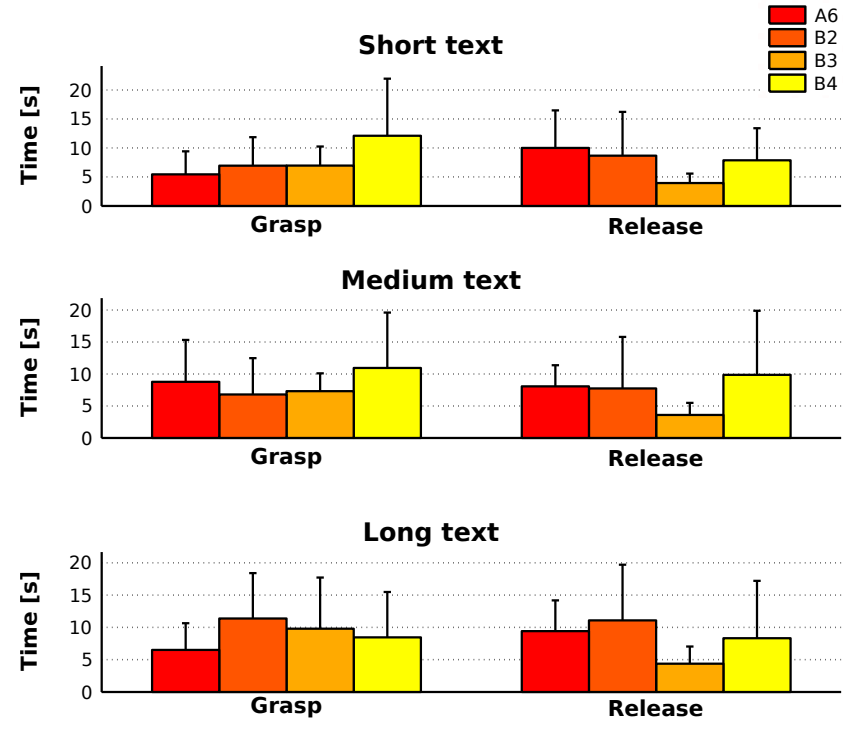

Fig. 3. The figure shows the average and standard deviation of the time required to the subjects to deliver mental commands to the BCI to grasp or release the pen. The three panels depict the grasp time and the release time for the three types of texts (short, medium, long). Although the subjects were not following a cue-based protocol, they were asked to grasp and release the pen as quickly as possible.

successful in writing, all of them reported that the task was slightly more involving than the natural dynamic tripod grasp. The different individual strategies, together with the low compliant grasp, surely contributed to the duration of the handwriting task.

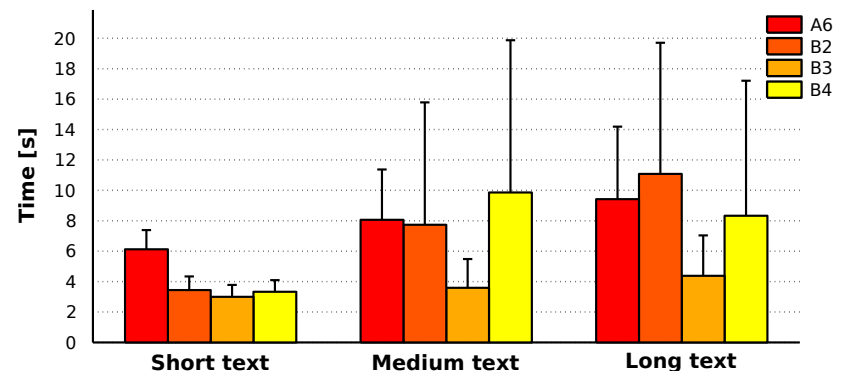

Fig. 4. The figure shows the average and standard deviation of the duration of the handwriting task computed across all the trials for the three types of texts (short, medium and long).

As previously said, a trial in which the subject failed to grasp the pen, released it prematurely and/or not in the pen holder was considered as failed. Although all the subjects were able to control the BCI with high accuracy, a certain number of unintended commands were delivered during the recordings, causing the premature release of the pen. We refer to these errors as sporadic errors. On the other hand, subject A6 and B4 entered short periods in which BCI control was difficult due to fatigue and lack of attention. This led to the sequential delivery of a certain number of unintended commands, referred as error bursts (Figure 5). For the burst case, a sequence of errors is reported as a single one, so as to include in the performance evaluation metrics those periods in which the subjects were not able to 


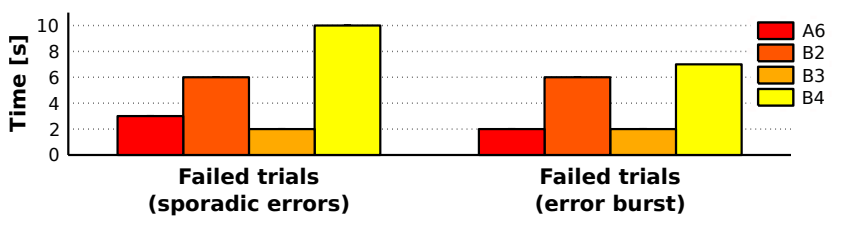

Fig. 5. The plot on the left shows the total number of failed trials due to sporadic errors. The plot on the right depicts the total number of failed trials due to error bursts. As mentioned in the text, error bursts are caused by fatigue and lack of attention. Under these circumstances, the subjects entered periods in which $\mathrm{BCI}$ control is troublesome, and a certain number of sequential errors were made. For this very last reason, errors bursts are reported as a single error.

momentarily control the BCI. Under this perspective, only novel subjects B2 and B4 were significantly affected by error bursts, while experience subjects A6 and B3 suffered minimally such error bursts. It is also worth noting that the erroneous commands were delivered uniformly across the whole recordings.

\section{CONCLUSIONS}

In this paper we have shown how healthy subjects can operate a non-invasive asynchronous BCI for controlling a FES neuroprosthesis and manipulate objects to carry out daily tasks in ecological conditions. Both, experienced and novel subjects proved to be able to deliver mental commands with high accuracy and speed. Interestingly, the novel subject A6 reached performances very close to B3's, our more experienced subject. This illustrates the benefit of utilizing a robust machine learning approach to select discriminant user-specfic features and to recognize the subject's intent.

Our neuroprosthetic approach relies on a natural interaction paradigm, where subjects delivers congruent MI commands (i.e., they imagining a movement of the same hand they control through FES, the dominant hand in the experiments reported here). In an attempt to evaluate our neuroprosthetic approach in ecological conditions, the experimental design does not impose constraints on how to perform the task, apart from achieving it as quickly as possible. Figure 4 shows how the time required to write the same text varies across the subject, thus clearly illustrating how subjects were performing the handwriting task with different strategies (e.g., speeds, styles). Furthermore, we have tested our approach in a common daily task such as handwriting, which requires the user to split his/her attention to multitask between BCI control, reaching, and the primary handwriting task itself. Therefore, it constitutes a proof of concept that non-invasive neuroprostheses can be effectively deployed in ecological activities requiring a high degree of interaction with the environment.

BCI control and task execution are intimately coupled. In fact, the subjects were not delivering any mental command to the FES either while writing, to release the pen, or while waiting for the next text and placing the hand over the pen holder, to grasp in the void. Importantly, the subjects were instructed not to perform MI tasks in any of these two periods (i.e., they could not engage in MI of the opposite BCI task to avoid triggering the FES). Similarly, the subjects were performing actions while delivering, or preparing to deliver, mental commands. For example, many subjects initiated MI and the reaching task simultaneously.

The very low number of erroneous trials during the experiments reported in Figure 5 illustrates how subjects were able to deliver commands just when they intended to do so; as well as to deliver, or not to deliver, commands while splitting their attention between the BCI and the task or the environment. The reason for the delivery of unintended commands has to be found in the nature of the classifier embedded in the BC that discriminates between different mental commands. In fact, discriminant classifiers, such as the Gaussian, are designed to separate a known number of mental tasks and cannot explicitly handle arbitrary patterns as those generated during those periods in which the subject does not intend to control the BCI. To further facilitate natural manipulation and interaction with the environment, we will extend the work reported here with our approach to support idle states so that users can deliver commands only when they wish to do so [10].

As mentioned before, we have developed a passive hand orthosis equipped with a mechanical lock that supports and synchronizes the movement of the fingers driven by FES [9]. More importantly, once activated, the lock holds the fingers in the desired position (e.g., flexed in a grasp posture) without the need for continuous stimulation. This is a critical feature for a neuroprosthesis as it allows long-term use and avoids possible electrical and/or sensory interference in the EEG. As a next step, we will combine the natural neuroprosthetic approach described in this paper with such a passive hand orthosis.

\section{REFERENCES}

[1] K. Anderson, "Targeting recovery: Priorities of the spinal cord-injured population," Neurotrauma, vol. 21, pp. 1371-1383, 2004.

[2] V. Hentz and C. Le Clercq, Eds., Surgical Rehabilitation of the Upper Limb in Tetraplegia. W. B. Saunders Ltd., 2002.

[3] B. Blankertz, M. Krauledat, D. Dornhege, J. Williamson, R. MurraySmith, and K.-R. Müller, "A note on brain actuated spelling with the Berlin brain-computer interface," in Proc. HCI Int., 2007.

[4] J. d. R. Millán, F. Galán, D. Vanhooydonck, E. Lew, J. Philips, and M. Nuttin, "Asynchronous non-invasive brain-actuated control of an intelligent wheelchair," in Proc. 31st Annual Int. Conf. IEEE Eng. Med. Biol. Soc., 2009, pp. 3361-3364.

[5] G. Müller-Putz, R. Scherer, G. Pfurtscheller, and R. Rupp, "EEG-based neuroprosthesis control: A step towards clinical practice," Neurosci. Lett, vol. 382, pp. 169-174, 2005.

[6] — "Brain-computer interfaces for control of neuroprostheses: From synchronous to asynchronous mode of operation," Biomed. Technik, vol. 51, pp. 57-63, 2006.

[7] J. d. R. Millán, F. Renkens, J. Mouriño, and W. Gerstner, "Noninvasive brain-actuated control of a mobile robot by human EEG," IEEE Trans. Biomed. Eng., vol. 51, pp. 1026-1033, 2004.

[8] J. d. R. Millán, P. Ferrez, F. Galán, E. Lew, and R. Chavarriaga, "Noninvasive brain-machine interaction," Int. J. Pattern Recognit. Artif. Intell., vol. 22, pp. 959-972, 2008.

[9] R. Leeb, M. Gubler, M. Tavella, H. Miller, and J. d. R. Millán, "On the road to a neuroprosthetic hand: A novel hand grasp orthosis based on functional electrical stimulation," Proc. IEEE-EMBC conference, accepted 2010.

[10] M. Tavella, S. Perdikis, R. Leeb, and J. d. R. Millán, "Non-intentional control for asynchronous BCI: A statistical approach," in Proc. 4th Int. BCI Meeting, 2010. 